\title{
Synergistic antitumor effect with indoleamine 2,3-dioxygenase inhibition and temozolomide in a murine glioma model
}

\author{
Mitsuto Hanihara, MD, PhD, ${ }^{1}$ Tomoyuki Kawataki, MD, PhD, ${ }^{1}$ Kyoko Oh-Oka, PhD, ${ }^{2}$ \\ Kentaro Mitsuka, MD, PhD, ${ }^{1}$ Atsuhito Nakao, MD, PhD, ${ }^{2}$ and Hiroyuki Kinouchi, MD, PhD'1 \\ Departments of ${ }^{1}$ Neurosurgery and ${ }^{2}$ Immunology, Faculty of Medicine, University of Yamanashi, Chuo, Yamanashi, Japan
}

OBJECTIVE Indoleamine 2,3-dioxygenase (IDO), a key enzyme of tryptophan (Trp) metabolism, is involved in tumorderived immune suppression through depletion of Trp and accumulation of the metabolite kynurenine, resulting in inactivation of natural killer cells and generation of regulatory T cells (Tregs). It has been reported that high expression of IDO in cancer cells is associated with suppression of the antitumor immune response and is consistent with a poor prognosis. Thus, IDO may be a therapeutic target for malignant cancer. The authors have recently shown that IDO expression is markedly increased in human glioblastoma and secondary glioblastoma with malignant change, suggesting that IDO targeting may also have therapeutic potential for patients with glioma. The aim of this study was to investigate the antitumor effect of IDO inhibition and to examine the synergistic function of IDO inhibitor and temozolomide (TMZ) in a murine glioma model.

METHODS Murine glioma GL261 cells and human glioma U87 cells were included in this study. The authors used 3 mouse models to study glioma cell growth: 1) a subcutaneous ectopic model, 2) a syngeneic intracranial orthotopic model, and 3) an allogenic intracranial orthotopic model. IDO inhibition was achieved via knockdown of IDO in GL261 cells using short hairpin RNA (shRNA) and through oral administration of the IDO inhibitor, 1-methyl---tryptophan (1-MT). Tumor volume in the subcutaneous model and survival time in the intracranial model were evaluated.

RESULTS In the subcutaneous model, oral administration of 1-MT significantly suppressed tumor growth, and synergistic antitumor effects of 1-MT and TMZ were observed $(p<0.01)$. Mice containing intracranially inoculated IDO knockdown cells had a significantly longer survival period as compared with control mice $(p<0.01)$.

CONCLUSIONS These results suggest that IDO expression is implicated in immunosuppression and tumor progression in glioma cells. Therefore, combining IDO inhibition with standard TMZ treatment could be an encouraging therapeutic strategy for patients with malignant glioma.

http://thejns.org/doi/abs/10.3171/2015.5.JNS141901

KEY WORDS indoleamine 2,3-dioxygenase; glioma; 1-methyl---tryptophan; temozolomide; oncology

$\mathrm{M}$ ALIGNANT glioma is the most common brain tumor in adults; it has an aggressive lethal nature and a median survival of only 14 months, despite the standard established therapy of maximum resection followed by radiation and chemotherapy. Several immunotherapies, such as dendritic cell therapy, have been evaluated as new adjuvant approaches. However, the efficacy of immunotherapy for patients with malignant glioma is limited for several reasons, including the anatomical iso- lation of the central nervous system by the blood-brain barrier, the absence of a lymphatic drainage system, and the ability of glioma cells to escape recognition by the immune system. Recent studies suggest that indoleamine 2,3-dioxygenase (IDO), the initial rate-limiting enzyme in tryptophan (Trp) metabolism, may be involved in such tumor-induced escape from immunosurveillance, showing an immunosuppressive function. ${ }^{14,27}$ IDO is expressed in various human cancers such as malignant melanoma, ovar-

ABBREVIATIONS FACS = fluorescence-activated cell sorter; GAPDH = glyceraldehyde-3-phosphate dehydrogenase; IDO = indoleamine 2,3-dioxygenase; IDO-KD = IDO-knockdown; INF- $\gamma=$ interferon- $\gamma$; PBS = phosphate-buffered saline; RT-PCR = real-time polymerase chain reaction; SEM = standard error of the mean; shRNA = short hairpin RNA; TMZ = temozolomide; Treg = regulatory T cell; Trp = tryptophan; 1-MT = 1-methyl---tryptophan.

SUBMITTED August 15, 2014. ACCEPTED May 21, 2015.

INCLUDE WHEN CITING Published online December 4, 2015; DOI: 10.3171/2015.5.JNS141901. 
ian cancer, and colorectal cancer, and the IDO expression level is clinically correlated with tumor progression and poor clinical outcomes..$^{20,24,26}$ Expression of IDO causes the local depletion of Trp and accumulation of toxic Trp catabolites, leading to growth arrest and apoptosis of alloreactive T cells or natural killer cells. ${ }^{3-7,10-12,15,25}$

We have already reported that IDO is highly expressed in human glioblastoma tissue compared with low-grade glioma and that IDO expression is increased in the secondary glioblastoma tissue with malignant transformation..$^{13}$ In addition, a recent study showed that serum Trp levels were significantly decreased in patients with glioblastoma compared with healthy controls. ${ }^{18}$ These findings suggest that Trp catabolism is definitely associated with glioma progression, and IDO inhibition seems to be a promising target for cancer treatment. In other cancers, several studies have reported that IDO inhibition has an antitumor effect in vivo., ${ }^{9,14,27,30,32}$ However, it remains to be elucidated if IDO inhibition can attenuate glioma growth in animal models.

Temozolomide (TMZ), which causes alkylation of DNA, subsequent double-strand breaks, and apoptosis, is a standard chemotherapeutic agent for malignant glioma. Although TMZ improves the prognosis for patients with glioma, its clinical efficacy is partial and limited. Many adjuvant therapies have been evaluated for use with TMZ, but no further improvement in prognosis has been reported. Although the IDO inhibitor 1-methyl-L-tryptophan (1MT) was ineffective as a single therapeutic agent in mouse models of transplantable breast cancer, the combination of 1-MT with various cytotoxic chemotherapeutic drugs, including alkylating agents, showed a synergistic effect in tumor regression. ${ }^{14}$ Therefore, combining TMZ and IDO inhibition could be an effective therapeutic strategy for patients with malignant glioma.

The present study examined IDO inhibition and the synergistic effect of IDO inhibition plus TMZ in a murine glioma syngeneic model.

\section{Methods}

\section{Cell Lines and Reagents}

The murine glioma cell line GL261 was kindly provided by Dr. Toshiki Yamasaki, Department of Neurosurgery, Shimane University Faculty of Medicine (Izumo, Shimane, Japan). The human glioma cell line U87 was obtained from American Type Culture Collection (ATCC). These cell lines were cultured and maintained in RPMI 1640 medium (Sigma-Aldrich) with $10 \%$ fetal bovine serum, $2 \mathrm{mM}$ glutamine, $100 \mathrm{U} / \mathrm{ml}$ penicillin, and $100 \mu \mathrm{g} /$ $\mathrm{ml}$ streptomycin in a humidified incubator at $37^{\circ} \mathrm{C}$ under $5 \% \mathrm{CO}_{2}$. GL261 cells can produce aggressive tumors in syngeneic mice, ${ }^{1,18}$ and U87 cells express IDO and are established as an intracranial model in xenograft mice.

\section{Quantitative Real-Time Polymerase Chain Reaction}

Quantitative real-time polymerase chain reaction (RTPCR) analysis was performed using the AB7300 RealTime PCR system (Applied Biosystems) according to the manufacturer's instructions, using primers and probes for IDO1 (Mm00492586_m1; Applied Biosystems) and glyceraldehyde-3-phosphate dehydrogenase (GAPDH; Mm99999915_g1; Applied Biosystems), as previously described. ${ }^{17}$ The ratio of target gene expression to GAPDH was calculated, and the relative expression levels of each gene were shown.

\section{IDO Short Hairpin RNA Knockdown}

Prepackaged lentiviral particles were purchased from a commercial provider (Santa Cruz Biotechnology) and encoded either a nontargeting short hairpin (sh)RNA (negative shRNA, sc-108060) or target-specific sequences for mouse IDO1 shRNA (IDO shRNA plasmid, sc-41530SH). For viral infection of GL261 cells, the regular medium was replaced with transfection medium (sc-108062). GL261 cells were then exposed to lentiviruses for 48 hours. Since the lentiviral shRNA particles also encode a puromycin resistance gene used for transduction selection, the cells were then washed and grown in culture medium containing $5 \mu \mathrm{g} / \mathrm{ml}$ puromycin dihydrochloride (P9620, Sigma-Aldrich) for an additional 72 hours. GL261 cells were allowed to recover and proliferate for at least 1 week before any experimental procedures were performed. After puromycin screening, individual colonies were chosen and examined for expression of endogenous IDO. Knockdown efficiency was analyzed by quantitative RT-PCR.

\section{Cell Viability Assay}

GL261 cells $\left(5 \times 10^{3}\right)$ were seeded onto 96-well culture plates and incubated in $100 \mu \mathrm{l}$ complete medium for 48 hours in the absence or presence of 1-MT $(500 \mu \mathrm{M})$ and TMZ $(200 \mu \mathrm{M})$. Cell viability was determined by trypan blue dye exclusion and the water-soluble tetrazolium assay using the TetraColor ONE kit (Seikagaku Corporation) according to the manufacturer's instructions.

\section{Flow Cytometry}

Spleen cells were obtained from mice harboring grafted glioma cells for 14 days. After the removal of red blood cells, spleen cells were probed with phycoerythrin-conjugated anti-mouse CD8 antibody, fluorescein isothiocyanate-conjugated anti-mouse CD3 antibody, and peridinin chlorophyll protein complex-conjugated anti-mouse CD4 antibody (eBioscience, Inc.). Cellular staining was analyzed using a FACSCalibur system (BD Biosciences, Inc.) with the Cell Quest program (BD Biosciences, Inc.). Negative controls consisted of isotype-matched, directly conjugated, nonspecific antibodies.

\section{Tissue Specimens and Immunohistochemistry}

Murine glioma tissue specimens were fixed in a $10 \%$ formalin solution, processed, and embedded in paraffin. The sections $(4 \mu \mathrm{m})$ were stained with hematoxylin and eosin or processed for immunohistochemical staining for IDO. Sections were deparaffinized in xylene followed by hydration using a graded-alcohol series, and the endogenous peroxidase activity was blocked by incubation with $2 \% \mathrm{H}_{2} \mathrm{O}_{2}$ in methanol. Sections were microwaved in 10 $\mathrm{mM}$ citrate buffer ( $\mathrm{pH}$ 6.0) for 10 minutes at $95^{\circ} \mathrm{C}$ for antigen retrieval. Sections were incubated with IDO primary antibody (1:50 dilution, Chemicon MAB5412, clone 10.1, EMD Millipore) or mouse IgG as a negative control 
(N1698, Dako) overnight at $4^{\circ} \mathrm{C}$. Next, the streptavidinlinked secondary antibody (LSAB+ Kit, Dako) was applied for 30 minutes, followed by streptavidin peroxidase for 15 minutes. After incubation in the substrate chromogen solution for 5 minutes, the slides were counterstained with hematoxylin for 3 minutes.

\section{Animal Experiments}

Animals were treated in accordance with the Yamanashi University guidelines, and the animal experimental protocols were approved by Yamanashi University's Administrative Panel on Laboratory Animal Models.

Five-week-old female C57BL/6 mice and athymic (nu/ nu) Balb/c mice were purchased from CLEA Japan, Inc. For the subcutaneous model, $10^{6}$ GL261 cells were injected into the lower right flank of each C57BL/6 mouse ( $\mathrm{n}=$ 10 ; all $\mathrm{n}$ values represent the number of mice per group). For the intracranial model, C57BL/6 and Balb/c mice were anesthetized by intraperitoneal injection of ketamine $(80-100 \mathrm{mg} / \mathrm{kg})$ and xylazine $(10 \mathrm{mg} / \mathrm{kg})$ and were immobilized in a stereotactic frame (NARISHIGE Type SR$5 \mathrm{~N}$ ) for tumor implantation. A 1-mm-diameter bur hole was drilled $3 \mathrm{~mm}$ lateral and $1 \mathrm{~mm}$ anterior to the bregma. With antiseptic technique, $5 \times 10^{5}$ GL261 cells (suspended in $3 \mu \mathrm{l}$ phosphate-buffered saline [PBS]) were stereotactically implanted using a Hamilton syringe at a depth of 3.5 $\mathrm{mm}$ from the dura mater ( $\mathrm{n}=6$ to 15 ). Additionally, human glioma U87 cells were intracranially implanted into the brains of Balb/c mice $(n=7)$, and the mice were then treated with an oral administration of 1-MT. Survival time was compared with the control group.

Mice from both the subcutaneous $(\mathrm{n}=10)$ and intracranial $(n=15)$ models were treated with an intraperitoneal injection of either TMZ (800 $\mu \mathrm{g}$ in $100 \mu \mathrm{l} \mathrm{PBS})$ or PBS alone (100 $\mu \mathrm{l}$; controls) every 2 days for 4 cycles, beginning on Day 7. The injections were combined with or without daily oral 1-MT treatment $(1 \mathrm{mg} / \mathrm{ml})$.

Tumor volume was measured with calipers and calculated using the following formula: $0.5 \times$ longest diameter $\times$ shortest diameter. ${ }^{2}$ After 14 days, the mice were killed, and the tumors and spleens were harvested. In the intracranial model, mice were observed daily and killed when they became ill or moribund. A total of 146 mice were killed among all of the experiments.

\section{Statistical Analysis}

Quantitative data were analyzed using the statistics software SPSS 15.0. Statistical comparisons were performed using a 2-tailed Student t-test. Survival time was calculated using the Kaplan-Meier method in each treated group so as to compare with the log-rank test.

A p value less than 0.05 was considered significant. All data are expressed as the mean \pm standard error of the mean (SEM).

\section{Results}

\section{Expression of IDO In Vitro}

Interferon- $\gamma(\mathrm{INF}-\gamma)$ is a well-known inducer of the mouse Idol gene in various cancer cell lines. We examined INF- $\gamma$-induced expression of Idol in GL261 cells. Idol ex- pression was very low in untreated GL261 cells and was greatly increased by stimulation with INF- $\gamma$ (Fig. 1 left). We established GL261 stable IDO-knockdown (IDO-KD) cells and control GL261 cells. Knockdown efficiency was analyzed by measuring Idol mRNA expression in IDO$\mathrm{KD}$ cells. The Idol expression level (relative to GAPDH expression) in IDO-KD cells decreased to $32 \%$ of control levels after INF- $\gamma$ stimulation (Fig. 1 right).

\section{Effect of IDO Inhibition on Cell Viability}

After confirming Idol knockdown efficiency in IDOKD cells, we evaluated cell proliferation in vitro. There was no significant difference in cell viability between IDO-KD cells and control GL261 cells (Fig. 2 left). Also, no significant difference was found in cell viability between the cells treated with TMZ only and cells treated with TMZ plus 1-MT (Fig. 2 right). These findings indicate that Idol expression level and the addition of 1-MT into the culture medium did not affect cell viability in vitro.

\section{Immunohistochemical Expression of IDO In Vivo}

Murine glioma tissue consisting of IDO-KD and control GL261 cells was fixed with formalin and embedded with paraffin on Day 14 after the cells were grafted. IDO expression was examined by immunohistochemistry in these murine glioma tissues. Positive immunoreactivity was confirmed in all tumor tissues. IDO immunoreactivity was detected in the murine glioma cell cytoplasm, predominantly at the periphery of the control GL261 glioma graft (Fig. 3A and B). IDO immunoreactivity was relatively weaker in the tissue around the IDO-KD glioma graft (Fig. 3C and D).

\section{Effect of IDO Inhibition and Synergistic Effect of 1-MT With TMZ on Tumor Volume in the Subcutaneous Model}

Compared with control GL261 cells, growth of IDO-
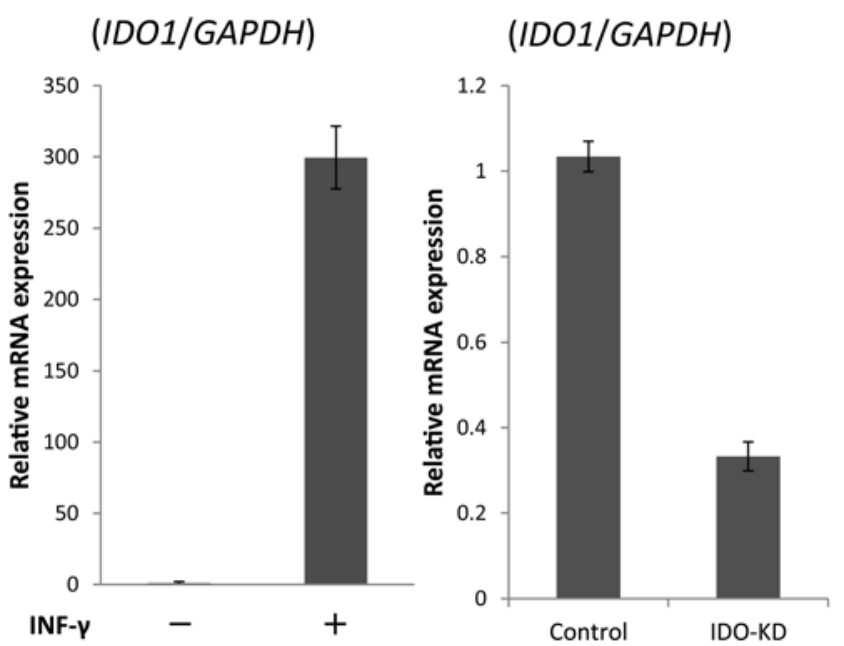

FIG. 1. Left: RT-PCR of $I d 01$ mRNA expression in the murine glioma cell line GL261 with or without INF- $\gamma(n=3$ per group). INF- $\gamma$ stimulation dramatically increased Ido1 mRNA expression in GL261 cells. Right: IDO knockdown efficiency was analyzed by RT-PCR in control and IDOKD cells stimulated with INF- $\gamma$ ( $n=3$ per group). 

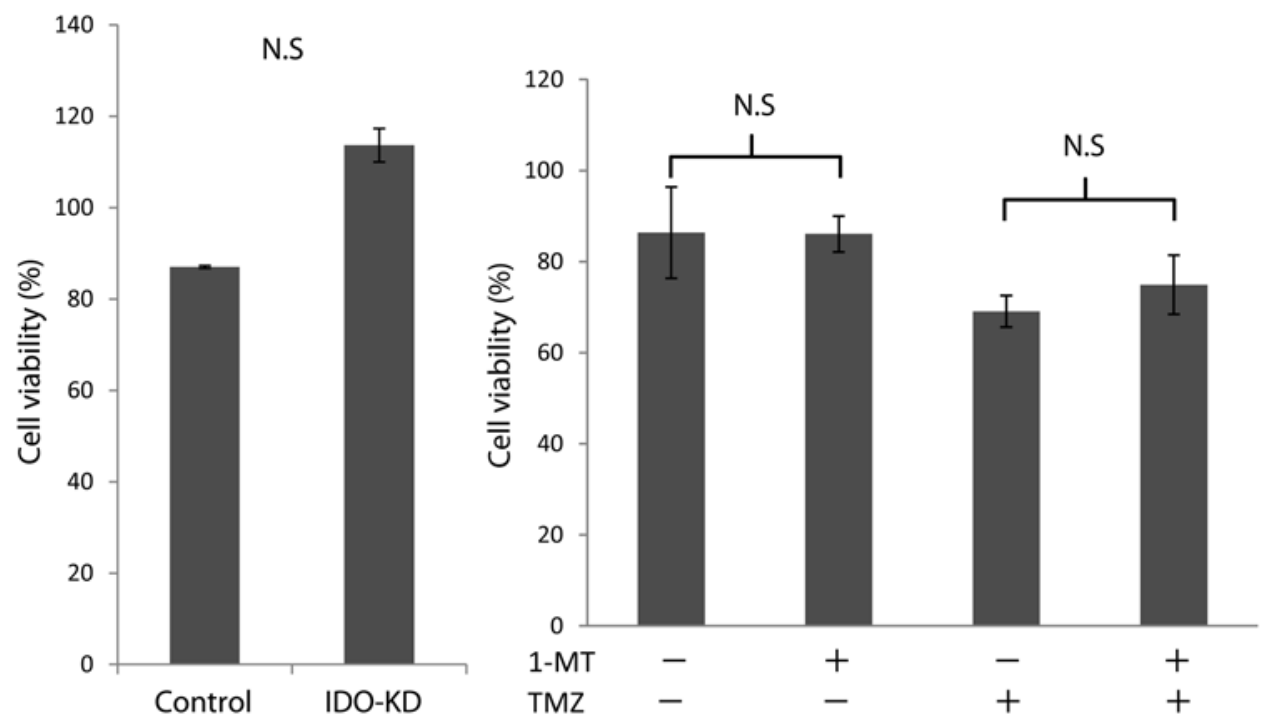

FIG. 2. Graphs showing the effect of IDO-KD (left) and 1-MT plus TMZ treatment (right) on murine glioma GL261 cell viability. GL261 cells $\left(5 \times 10^{3}\right)$ were seeded onto 96 -well culture plates and incubated with $0.1 \mathrm{ml}$ complete medium in the absence or presence of 1-MT $(500 \mu \mathrm{M})$ and TMZ $(200 \mu \mathrm{M})$. Cell viability was determined using the water-soluble tetrazolium assay. N.S = not significant.

KD cells was strongly inhibited during the initial 2 weeks after inoculation (relative mean tumor volume compared to control was $5 \%, \mathrm{p}=0.008$; Fig. 4 upper). In addition, oral administration of 1-MT to the mice harboring syngeneic glioma cells suppressed tumor growth as compared with control vehicle (mean tumor volume was $46 \%$ relative to control, $p=0.005 ;$ Fig. 4 lower). Investigation of the synergistic effects of 1-MT and TMZ revealed that tumor growth was suppressed by treatment with 1-MT plus TMZ as compared to TMZ alone (mean tumor volume was $55 \%$ relative to TMZ alone, $\mathrm{p}=0.002$; Fig. 4 lower).

\section{Effect of IDO on In Vivo T Cell Population}

To determine the antitumor mechanism in the murine

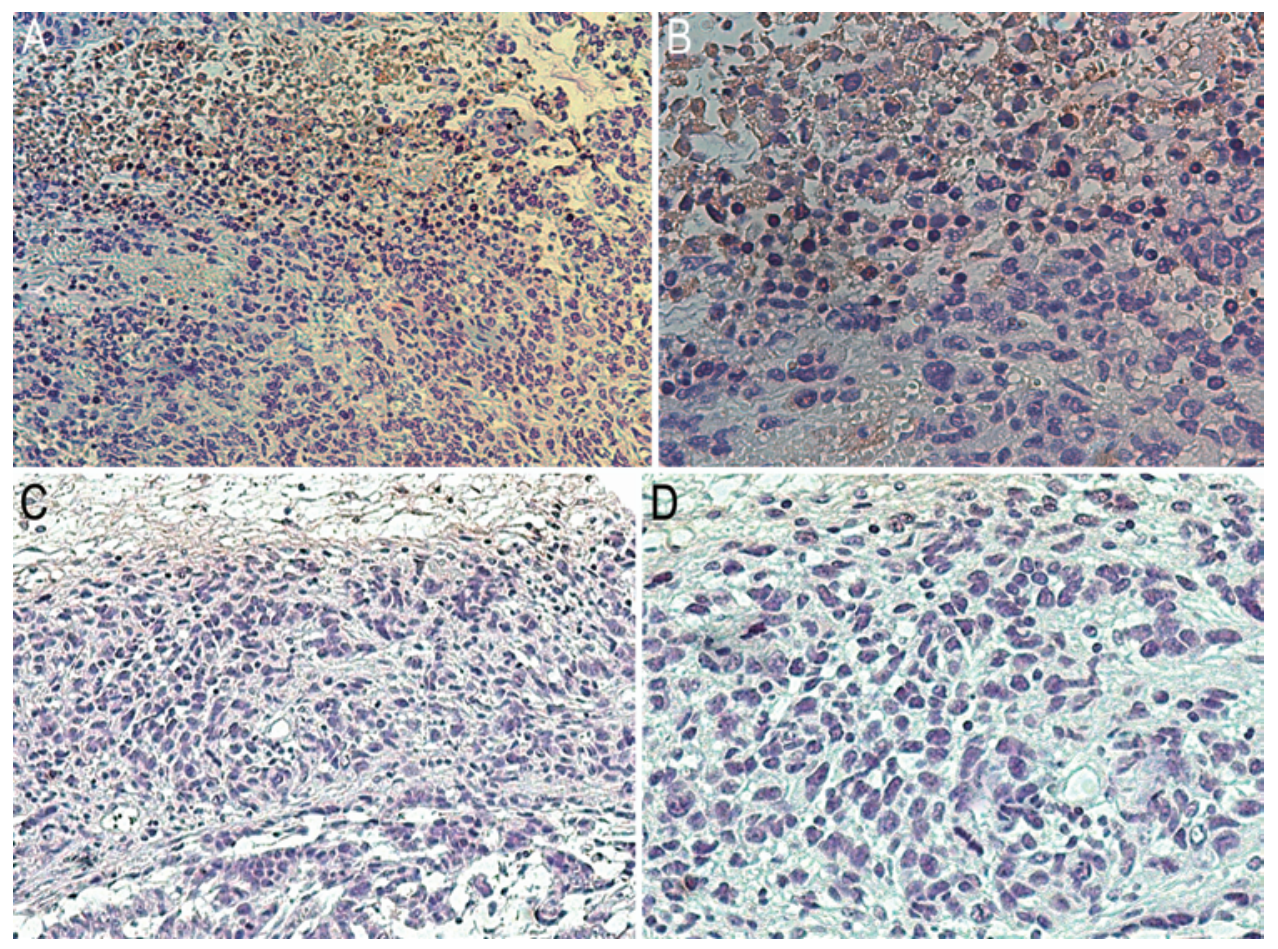

FIG. 3. Immunohistochemical analysis of IDO in a murine glioma syngeneic model. IDO staining of tissue from a C57BL/6 mouse implanted with GL261 glioma cells in the subcutaneous flank. IDO immunoreactivity was detected in the periphery of the tumor (A and B). Weak IDO expression is observed in IDO-KD of glioma cell graft tissue (C and D). Original magnification $\times 40$ (A and C) and $\times 400(B$ and $D)$. 
glioma model, we examined the effect of IDO inhibition on the T cell population. Fluorescence-activated cell sorter (FACS) analysis showed that the percentage of CD4+ T cells and CD8+ T cells in spleens taken from GL261 tumorbearing mice on Day 14 after inoculation was markedly increased in the IDO-KD group and in the 1-MT group compared with the control group ( $\mathrm{p}<0.005$; Fig. 5A-D).

\section{Effect of IDO Inhibition on Survival Rate in the Intracranial Model}

All control mice for the intracranial cell implantation procedure had become moribund and were killed between 3 and 5 weeks after intracranial inoculation. The mice implanted with IDO-KD glioma cells had significantly longer survival compared with the control group $(p=0.001)$ (Fig. 6A).

The survival rate was relatively higher in the 1-MT group than in the control group $(\mathrm{p}=0.117$; Fig. $6 \mathrm{~B})$. Additionally, the 1-MT plus TMZ group had a tendency toward a longer survival time than the TMZ-alone group $(\mathrm{p}=0.099)$. In the intracranial xenograft model using the human glioma cell line U87 in athymic (nude) mice, there was no statistically significant difference in survival time between the 1-MT group and controls (Fig. 6C).

\section{Discussion}

The present findings demonstrate that IDO inhibition significantly suppressed glioma cell growth in murine glioma xenograft models. IDO was inhibited by knockdown of Idol expression in cells by shRNA and by oral administration of the IDO inhibitor 1-MT in the subcutaneous and intracranial models. Although oral administration of 1-MT in the intracranial model tended to show an antitumor effect on survival, it did not reach statistical significance. However, inoculation of IDO-KD glioma cells in the intracranial model resulted in statistically significant increases in survival compared with the control mice. This study provided consistent evidence of the antitumor effect of IDO inhibition in an in vivo syngeneic glioma model.

In previous studies, IDO expression has led to poor outcomes in patients with several carcinomas. ${ }^{8}$ In glioma, we have reported that IDO was highly expressed in human glioblastoma tissue as compared with low-grade glioma, and IDO expression was increased in secondary glioblastoma tissue with malignant transformation..$^{13}$ Therefore, IDO could be a novel and reliable prognostic indicator of patient outcomes and may be a crucial therapeutic target for malignant glioma.

Given that IDO expression was induced by INF- $\gamma$, one could argue that highly expressed IDO reflects an immunosuppressive phenomenon that acts as a negative feedback mechanism against immune responses. However, in the present study, IDO inhibition via shRNA knockdown suppressed tumor growth by increasing the number of CD4+ and CD8+ T cells in the murine xenograft model. These results suggest that IDO derived from glioma cells suppressed the proliferation of effector cells such as helper $\mathrm{T}$ cells, macrophages, and killer T cells.

The mechanism of IDO-induced immune escape remains unclear. However, it has been suggested that local
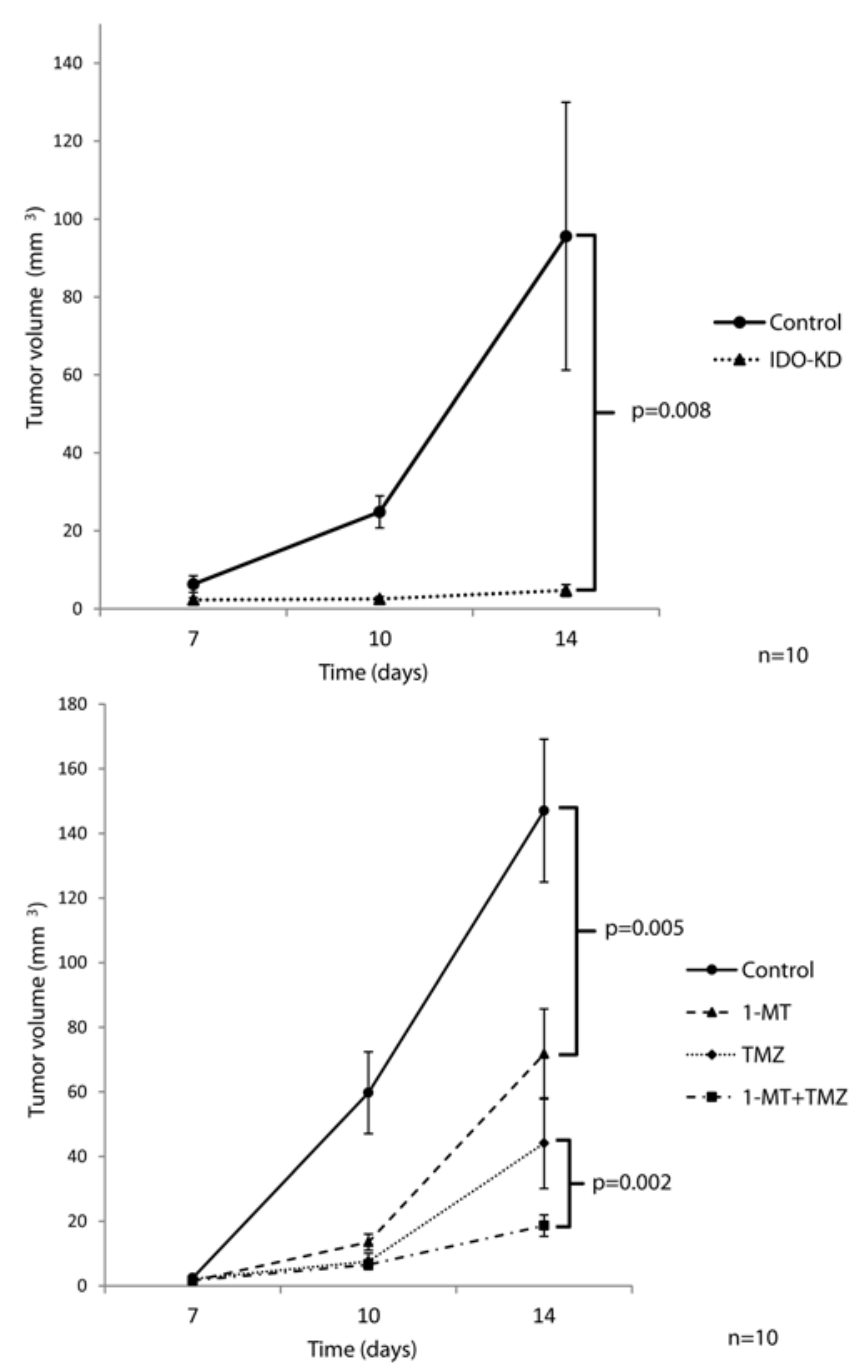

FIG. 4. Control or IDO-KD murine glioma GL261 cell grafts injected subcutaneously into the flank of $\mathrm{C} 57 \mathrm{BL} / 6$ mice were measured with metric calipers $(n=10)$, and tumor volume was calculated. IDO-KD cell growth was significantly inhibited compared with the control cells during the initial 2 weeks after injection (mean tumor volume was $5 \%$ relative to controls, $p=0.008$ ). Error bars indicate SEM (upper). Murine glioma GL261 cell grafts injected subcutaneously into the flank of C57BL/6 mice were monitored with metric calipers $(n=10)$, and tumor volume was calculated. Mice received either normal drinking water or water containing 1-MT (1 mg/ml) with or without intraperitoneal TMZ. Tumor growth was suppressed by 1-MT compared with control vehicle (mean tumor volume was $46 \%$ relative to controls, $p=0.005$ ). Tumor growth was suppressed by 1-MT plus TMZ compared with TMZ alone (mean tumor volume was $55 \%$ relative to $T M Z$ alone, $p=0.002$ ). Error bars indicate SEM (lower).

Trp metabolism is essential. Activation of IDO results in the depletion of Trp and the accumulation of downstream metabolites such as kynurenine. ${ }^{25}$ These biochemical changes cause cell cycle arrest of T cells at mid-G1 phase through general control of Eukaryotic Translation Initiation Factor 2-alpha Kinase 4 (EIF2AK4). ${ }^{16}$ Activation of EIF2AK4 downregulates most amino acid synthesis to adapt to starvation in the cell environment. Recent studies suggest that effector $\mathrm{T}$ cells are preferentially sensitive to the accumulation of kynurenine and other downstream 

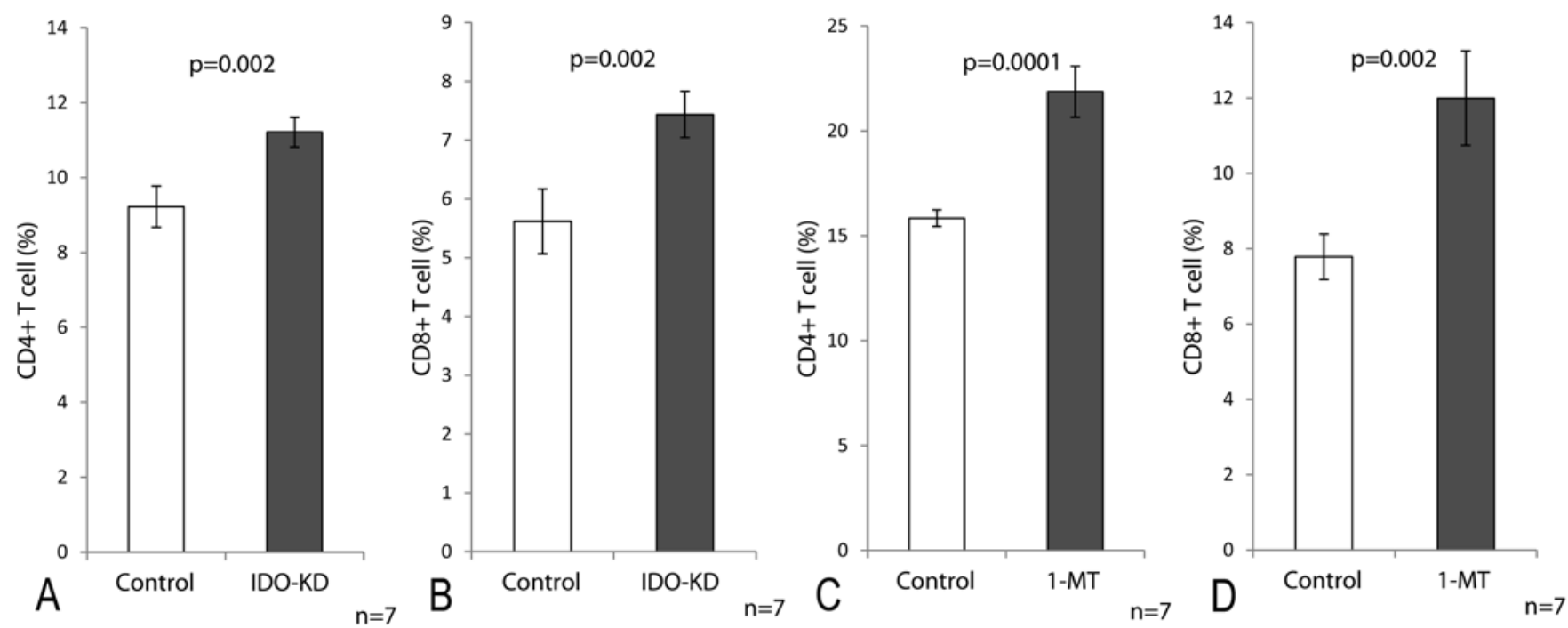

FIG. 5. Flow cytometric analysis of CD4+ and CD8+ T cells from the spleens of C57BL/6 mice on Day 14 after subcutaneous injection of GL261 cells. The percentages of CD4+ and CD8+ T cells in spleens were significantly increased in the IDO-KD group (A and $B$ ) and the 1-MT group (C and D) ( $n=7$ per group).
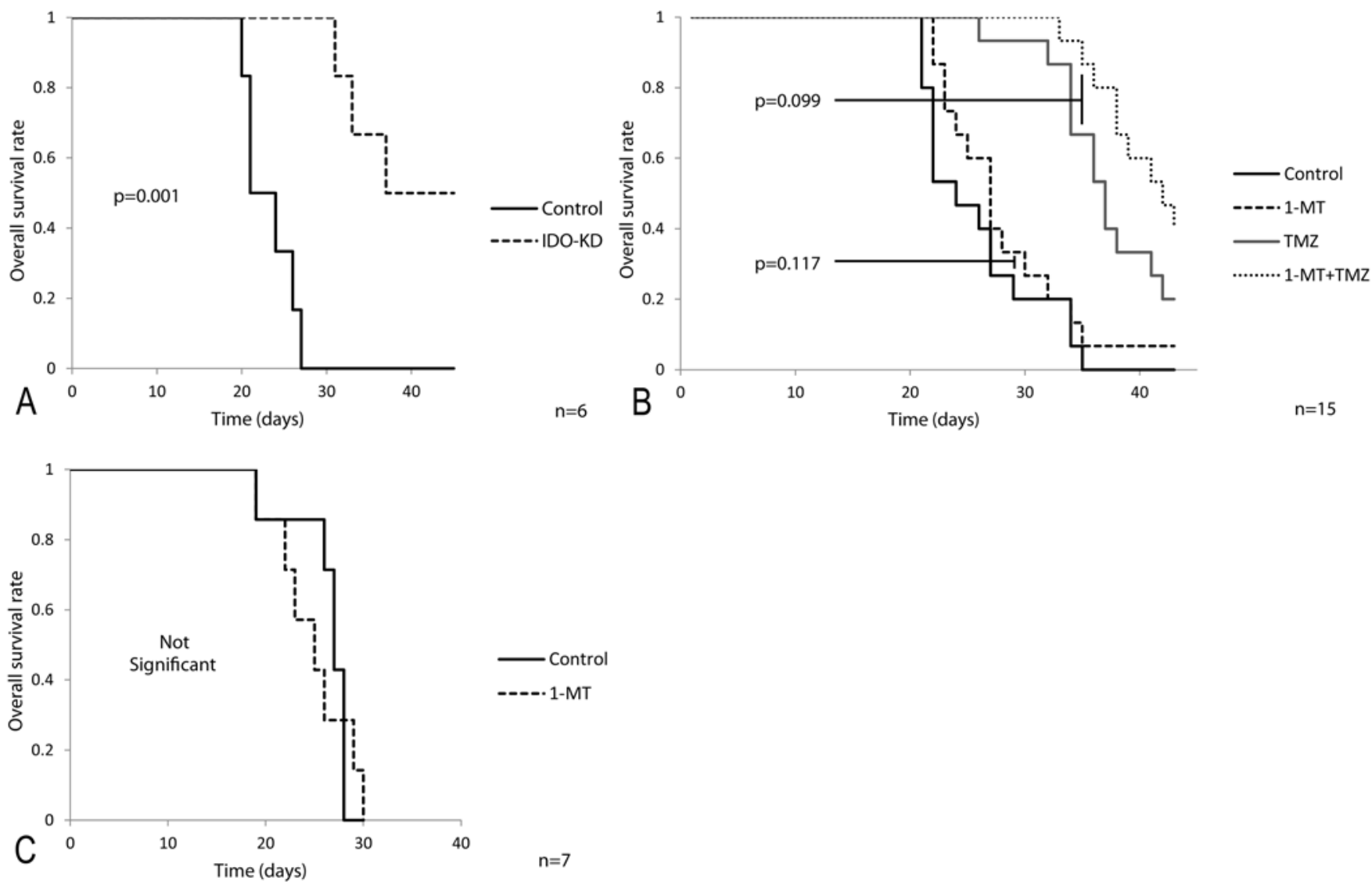

FIG. 6. A significantly higher survival rate was observed in IDO-KD cells compared with control GL261 cells $(p=0.001$; A). Orthotopic GL261 cells were implanted stereotactically into the right frontal lobes of syngeneic C57BL/6 host mice, and Kaplan-Meier survival plots are shown. The survival rate was relatively higher in the 1-MT group compared with the control group $(p=0.117)$ and in the 1-MT plus TMZ group compared with TMZ alone $(p=0.099 ; B)$. In the orthotopic U87 xenograft model, a statistically significant difference was not observed between the control group and the 1-MT group (C). 
catabolites generated by the IDO pathway. ${ }^{19}$ Our results in the murine glioma syngeneic model support these published findings.

Additionally, the present study shows for the first time a synergistic antitumor effect of administering 1-MT plus TMZ in a subcutaneous mouse model. TMZ is an alkylating agent that methylates the $\mathrm{O} 6$ position of guanine, causing cross-linking of double-stranded DNA. ${ }^{2}$ The DNA damage results in calcium-dependent apoptosis and autophagy, eventually leading to cell death. Although TMZ is used as a first-line agent for malignant glioma, its use as a single agent has only partial and limited efficacy. Therefore, many combination therapies with TMZ have been evaluated to increase the adjuvant effect, which is the primary strategy of novel treatments for malignant glioma. A phase I clinical trial of 1-MT has already been reported and has demonstrated good bioavailability with no treatment-related severe adverse events..$^{22}$ In addition, another phase I clinical trial that combined the use of an IDO inhibitor with docetaxel demonstrated encouraging antitumor activity and safety against solid metastatic tumors. ${ }^{21}$ Moreover, a phase I/II study investigating the combined use of 1-MT and TMZ for treatment of malignant brain tumors is ongoing in the United States. ${ }^{31}$

It has been reported that combining 1-MT with alkylating chemodrugs such as cyclophosphamide and cisplatin resulted in synergistically regressed tumors in mouse models of transplantable breast cancer. ${ }^{14}$ Although the mechanism of these synergistic effects is not fully understood, it was speculated that regulatory T cells (Tregs) play a dominant role. Treg populations are another important factor for acquiring immunological tolerance of a tumor in that recruitment of Tregs to the surrounding tumor tissue leads to tumor growth. ${ }^{28}$ IDO causes the induction of Tregs via its metabolite kynurenine, which acts on the aryl hydrocarbon receptor and induces the synthesis of the Treg-recruiting chemokine CCL22. ${ }^{29}$ TMZ leads to immune suppression by causing various forms of lymphopenia; however, it has been reported that TMZ-induced lymphopenia preferentially affects Tregs. ${ }^{23}$ Therefore, we speculate that the combination of TMZ plus 1-MT has a synergistic effect on Treg depletion. Further analyses are required to investigate the synergistic mechanism in greater detail.

In the syngeneic intracranial model, inhibition of IDO resulted in a relatively prolonged survival rate, especially when evaluated between 3 and 4 weeks after inoculation of glioma cells. However, the difference was not statistically significant. IDO-KD attenuated tumor growth and improved survival time to a greater degree than the oral administration of an IDO inhibitor. This discrepancy may be caused by the inhibitory efficiency of IDO in the brain. The IDO inhibitor 1-MT is not a lipophilic substance, and the permeability of 1-MT into brain tissue may be limited to areas of damaged blood-brain barrier that occur around the glioma tissue. Therefore, the creation of a more efficient IDO inhibitor that can easily pass through the bloodbrain barrier is highly desired.

In the xenograft model of athymic (nude) mice containing U87 human glioma cells, there was no significant difference in survival time between the IDO inhibitor group and controls. We speculate that the absence of $\mathrm{T}$ cells in nude mice is the cause of these results. T cells would play a key role in the IDO-induced immune escape in this murine glioma model. Another in vivo glioma study has shown that the survival advantage conferred by IDO deficiency was lost in T cell-deficient mice. ${ }^{28}$

One limitation of the present study is that the we did not examine the upregulation of immune responses following the inhibition of IDO. Additional studies examining the local immune response within or adjacent to the tumors is necessary.

\section{Conclusions}

These results suggest that IDO expression in glioma cells is implicated in immunosuppression and tumor progression; therefore, IDO inhibition with TMZ could be an encouraging therapeutic strategy for patients with malignant glioma.

\section{Acknowledgements}

We thank Grants-in-Aid for Scientific Research from the Japanese Ministry of Education, Culture, Sports, Science and Technology, Japan (20591709) for financial support.

\section{References}

1. Ausman JI, Shapiro WR, Rall DP: Studies on the chemotherapy of experimental brain tumors: development of an experimental model. Cancer Res 30:2394-2400, 1970

2. Baer JC, Freeman AA, Newlands ES, Watson AJ, Rafferty JA, Margison GP: Depletion of O6-alkylguanine-DNA alkyltransferase correlates with potentiation of temozolomide and CCNU toxicity in human tumour cells. Br J Cancer 67:1299-1302, 1993

3. Della Chiesa M, Carlomagno S, Frumento G, Balsamo M, Cantoni C, Conte R, et al: The tryptophan catabolite Lkynurenine inhibits the surface expression of NKp46- and NKG2D-activating receptors and regulates NK-cell function. Blood 108:4118-4125, 2006

4. El Andaloussi A, Lesniak MS: An increase in CD4+CD25+FOXP3+ regulatory T cells in tumor-infiltrating lymphocytes of human glioblastoma multiforme. Neuro Oncol 8:234-243, 2006

5. Fallarino F, Grohmann U, Vacca C, Bianchi R, Orabona C, Spreca A, et al: T cell apoptosis by tryptophan catabolism. Cell Death Differ 9:1069-1077, 2002

6. Fallarino F, Grohmann U, Vacca C, Orabona C, Spreca A, Fioretti MC, et al: T cell apoptosis by kynurenines. Adv Exp Med Biol 527:183-190, 2003

7. Frumento G, Rotondo R, Tonetti M, Damonte G, Benatti U, Ferrara GB: Tryptophan-derived catabolites are responsible for inhibition of $\mathrm{T}$ and natural killer cell proliferation induced by indoleamine 2,3-dioxygenase. J Exp Med 196:459-468, 2002

8. Godin-Ethier J, Hanafi LA, Piccirillo CA, Lapointe R: Indoleamine 2,3-dioxygenase expression in human cancers: clinical and immunologic perspectives. Clin Cancer Res 17:6985-6991, 2011

9. Hou DY, Muller AJ, Sharma MD, DuHadaway J, Banerjee T, Johnson M, et al: Inhibition of indoleamine 2,3-dioxygenase in dendritic cells by stereoisomers of 1-methyl-tryptophan correlates with antitumor responses. Cancer Res 67:792801,2007

10. Lee GK, Park HJ, Macleod M, Chandler P, Munn DH, Mellor AL: Tryptophan deprivation sensitizes activated T cells to 
apoptosis prior to cell division. Immunology 107:452-460, 2002

11. Liu X, Shin N, Koblish HK, Yang G, Wang Q, Wang K, et al: Selective inhibition of IDO1 effectively regulates mediators of antitumor immunity. Blood 115:3520-3530, 2010

12. Mellor AL, Munn DH: IDO expression by dendritic cells: tolerance and tryptophan catabolism. Nat Rev Immunol 4:762-774, 2004

13. Mitsuka K, Kawataki T, Satoh E, Asahara T, Horikoshi T, Kinouchi H: Expression of indoleamine 2,3-dioxygenase and correlation with pathological malignancy in gliomas. Neurosurgery 72:1031-1039, 2013

14. Muller AJ, DuHadaway JB, Donover PS, Sutanto-Ward E, Prendergast GC: Inhibition of indoleamine 2,3-dioxygenase, an immunoregulatory target of the cancer suppression gene Bin1, potentiates cancer chemotherapy. Nat Med 11:312-319, 2005

15. Munn DH, Shafizadeh E, Attwood JT, Bondarev I, Pashine A, Mellor AL: Inhibition of T cell proliferation by macrophage tryptophan catabolism. J Exp Med 189:1363-1372, 1999

16. Munn DH, Sharma MD, Baban B, Harding HP, Zhang Y, Ron $\mathrm{D}$, et al: GCN2 kinase in T cells mediates proliferative arrest and anergy induction in response to indoleamine 2,3-dioxygenase. Immunity 22:633-642, 2005

17. Nakamura Y, Miyata M, Ohba T, Ando T, Hatsushika K, Suenaga F, et al: Cigarette smoke extract induces thymic stromal lymphopoietin expression, leading to $\mathrm{T}(\mathrm{H}) 2$-type immune responses and airway inflammation. J Allergy Clin Immunol 122:1208-1214, 2008

18. Opitz CA, Litzenburger UM, Sahm F, Ott M, Tritschler I, Trump S, et al: An endogenous tumour-promoting ligand of the human aryl hydrocarbon receptor. Nature 478:197-203, 2011

19. Prendergast GC: Immune escape as a fundamental trait of cancer: focus on IDO. Oncogene 27:3889-3900, 2008

20. Riesenberg R, Weiler C, Spring O, Eder M, Buchner A, Popp $\mathrm{T}$, et al: Expression of indoleamine 2,3-dioxygenase in tumor endothelial cells correlates with long-term survival of patients with renal cell carcinoma. Clin Cancer Res 13:69937002, 2007

21. Soliman HH, Jackson E, Neuger T, Dees EC, Harvey RD, Han H, et al: A first in man phase I trial of the oral immunomodulator, indoximod, combined with docetaxel in patients with metastatic solid tumors. Oncotarget 5:8136-8146, 2014

22. Soliman HH, Neuger A, Noyes D, Vahanian NN, Link CJ, Munn D, et al: A phase I study of 1-methyl-D-tryptophan in patients with advanced malignancies. J Clin Oncol 30 Suppl:Abstr 2501, 2012

23. Su YB, Sohn S, Krown SE, Livingston PO, Wolchok JD, Quinn C, et al: Selective CD4+ lymphopenia in melanoma patients treated with temozolomide: a toxicity with therapeutic implications. J Clin Oncol 22:610-616, 2004

24. Takao M, Okamoto A, Nikaido T, Urashima M, Takakura $\mathrm{S}$, Saito M, et al: Increased synthesis of indoleamine-2,3dioxygenase protein is positively associated with impaired survival in patients with serous-type, but not with other types of, ovarian cancer. Oncol Rep 17:1333-1339, 2007

25. Terness P, Bauer TM, Röse L, Dufter C, Watzlik A, Simon H, et al: Inhibition of allogeneic $\mathrm{T}$ cell proliferation by indoleamine 2,3-dioxygenase-expressing dendritic cells: mediation of suppression by tryptophan metabolites. J Exp Med 196: $447-457,2002$

26. Urakawa H, Nishida Y, Nakashima H, Shimoyama Y, Nakamura S, Ishiguro N: Prognostic value of indoleamine 2,3-dioxygenase expression in high grade osteosarcoma. Clin Exp Metastasis 26:1005-1012, 2009

27. Uyttenhove C, Pilotte L, Théate I, Stroobant V, Colau D, Parmentier N, et al: Evidence for a tumoral immune resistance mechanism based on tryptophan degradation by indoleamine 2,3-dioxygenase. Nat Med 9:1269-1274, 2003

28. Wainwright DA, Balyasnikova IV, Chang AL, Ahmed AU, Moon KS, Auffinger B, et al: IDO expression in brain tumors increases the recruitment of regulatory $T$ cells and negatively impacts survival. Clin Cancer Res 18:6110-6121, 2012

29. Wainwright DA, Dey M, Chang A, Lesniak MS: Targeting Tregs in malignant brain cancer: overcoming IDO. Front Immunol 4:116, 2013

30. Yoshida N, Ino K, Ishida Y, Kajiyama H, Yamamoto E, Shibata K, et al: Overexpression of indoleamine 2,3-dioxygenase in human endometrial carcinoma cells induces rapid tumor growth in a mouse xenograft model. Clin Cancer Res 14:7251-7259, 2008

31. Zakharia Y, Johnson TS, Colman H, Vahanian NN, Link CJ, Kennedy E, et al: A phase I/II study of the combination of indoximod and temozolomide for adult patients with temozolomide-refractory primary malignant brain tumors. J Clin Oncol 32 Suppl:5s, 2014 (Abstract TPS2107)

32. Zheng X, Koropatnick J, Li M, Zhang X, Ling F, Ren X, et al: Reinstalling antitumor immunity by inhibiting tumor-derived immunosuppressive molecule IDO through RNA interference. J Immunol 177:5639-5646, 2006

\section{Disclosures}

The authors report no conflict of interest concerning the materials or methods used in this study or the findings specified in this paper.

\section{Author Contributions}

Conception and design: Kinouchi, Hanihara, Kawataki, Nakao. Drafting the article: Kinouchi, Hanihara. Critically revising the article: Mitsuka. Administrative/technical/material support: Ohoka.

\section{Correspondence}

Hiroyuki Kinouchi, Department of Neurosurgery, Faculty of Medicine, University of Yamanashi, 1110 Shimokato, Chuo, Yamanashi 409-3898, Japan. email: hkinouchi@yamanashi.ac.jp. 